\title{
Correlation between Patellar Tendon Mechanical Properties and Oxygenation Detection by Near Infrared Spectroscopy in Males
}

\author{
L. Calanni1 ${ }^{1 *}$, C. Zampella ${ }^{1 *}$, P. Micheletti ${ }^{2}$, D. Greco ${ }^{1}$, M. Negro ${ }^{1}$, G. D'Antona $^{1,2^{*}}$ \\ ${ }^{1}$ CRIAMS-Sport Medicine Centre, University of Pavia, Voghera, Pavia, Italy \\ 2 Department of Public Health, Experimental and Forensic Medicine, University of Pavia, Pavia, Italy \\ *These authors contributed equally to the work
}

\section{CORRESPONDING AUTHOR:}

Giuseppe D'Antona

Department of Public Health

Experimental and Forensic Medicine

Sport Medicine Centre Voghera

via Foscolo 13

27058 Voghera, Pavia, Italy

E-mail: gdantona@unipv.it

DOI:

10.32098/mltj.01.2021.06

LEVEL OF EVIDENCE: 3B

\section{ABBREVIATIONS:}

CSA: Cross-sectional area

sCSA: Cross-sectional area normalised to body

mass $2 / 3$

PT: Patellar tendon

RMVC: Ramped maximal voluntary contraction

NIR: Near Infrared

NIRS: Near Infrared Spectroscopy

$\mathrm{O}_{2} \mathrm{Hb}$ : Oxyhaemoglobin

$\mathrm{HHb}$ : Deoxyhaemoglobin

$\Delta \mathrm{HHb} \%$ : Deoxyhaemoglobin percentage change

tHb: Total haemoglobin volume

$\Delta \mathrm{tHb} \%$ : Total haemoglobin volume percentage

change

TOI: Tisssue Oxygen Index

\begin{abstract}
SUMMARY
Background. The purpose of this study was to determine the correlation between the patellar tendon mechanical properties and oxygenation in healthy men.

Methods. In two subsequent sessions, eight (mean age \pm SD: $27 \pm 4$ ) recreationally active, healthy males performed 4 -sec isometric knee extensions of the dominant leg at $20-80 \%$ of maximal voluntary contraction (MVC), maintaining a knee joint angle of $90^{\circ}$ of flexion.

In the first session, B-mode ultrasonography was used to record tendon length, cross-sectional area (CSA), and elongation, parameters needed to calculate stiffness. In the second session, tendon/peritendon hemodynamic parameters, namely total hemoglobin $\Delta \mathrm{tHb}, \Delta \mathrm{HHb}$ and tissue oxygenation index (TOI\% $=\mathrm{O}_{2} \mathrm{Hb} / \mathrm{tHb} \times 100$ ), as indicators of blood flow and oxygen extraction respectively were measured by near infrared spectroscopy (NIRS). Correlations between blood volume ( $\mathrm{tHB})$, tissue oxygenation and tendon mechanical properties were tested using Pearson's correlation coefficient (R).

Results. At all \% of MVCs, a reduction of $\mathrm{tHb}$ and TOI\%, and an increase of $\mathrm{HHb}$ were observed. At $80 \% \mathrm{MVC}, \Delta \mathrm{TOI} \%$ was negatively correlated with strain $(R=-0.64)$ and elongation $(R=-0.48)$, whereas a significant positive correlation was found with Young modulus $(\mathrm{R}=0.69)$ and stress $(\mathrm{R}=0.62)$; as regards blood volume, a significant negative correlation was found between elongation and $\Delta \mathrm{tHb}(\mathrm{R}=-0.44)$ and a positive correlation was found between stress and $\Delta \mathrm{tHb}(\mathrm{R}=0.4)$.

Conclusions. Patellar tendon mechanical properties and tendon/peritendon oxygen extraction and blood volume appears to be moderately correlated when studied by ultrasound and NIRS.
\end{abstract}

\section{KEY WORDS}

Haemoglobin; tendon; spectroscopy; stress-strain curves; oxygenation.

\section{BACKGROUND}

The tendon's blood circulation is poor, mostly peripheral (peritendinous sheet), and rises from the muscular belly and periosteum. The circulation is ensured by small and medium caliber arteries, followed by organized and disorganized capillary structures. A critical decrease in blood flow and, consequently, in oxygenation, may be potentially harm- ful and consequent ischemia/hypoxia may lead to tendon degeneration (tendinopathy). This condition is usually associated with overuse, particularly in athletes (1).

Hypoxia and reactive oxygen species production within the tendons and in their proximity $(2,3)$ could be considered the initiators of tendinopathy. In fact, hypoxia-related proteins are overexpressed early and regulate cell inflamma- 
tion and apoptosis. These processes promote a functionally significant shift in collagen matrix synthesis and expression (3). Biopsies from normal and ruptured tendons demonstrate that fibril interruption is associated with reduced type I collagen content (normally around $70 \%$ of the dry mass) together with a significantly increased proportion of type III collagen (the second most abundant under physiological conditions, tending to produce fibrils with less mechanical strength) (3). This composition change accounts for decreased resistance of tendon to tensile forces that may lead to subsequent rupture (4). Furthermore, decreasing tendon oxygenation may reflect local acidosis and increased intratendinous lactate accumulation that can contribute to the overall evolution of damage (5).

Although hypoxia is believed to trigger tissue damage, recent data highlights that neovascularization coexists with local pain, swelling, and size changes in tendinopathy, unlike insertional tendinopathy (6). Neovascularization and capillary blood flow increase have been demonstrated in Achilles tendon (AT), patellar tendon (PT), and in tennis elbow tendinopathy by using Color and/or Power Doppler ultrasound (US) (7). Overall, published data reveals that neovessels formation and subsequent changes in blood flow are part of the pathophysiological process leading to alterations at the tendon body along with its widening, disturbed collagen distribution and changes in cellularity (8).

Several tools are available for the assessment of the tendons structural and functional features, blood flow and oxygenation status, including conventional US, color Doppler/ Power Doppler US and backscattering-spectroscopy. Unlike conventional US for tendon assessment, that in the presence of tendinopathy reveals hypoechogenic texture within an enlarged tendon, color Doppler and Power Doppler US technologies are capable of identifying neovascularization because they allow visualisation of low-flow vessels. In its current routine application Power Doppler is of qualitative use only and does not allow to obtain data on tissue oxygen delivery and extraction, which can be assessed by backscattering spectroscopy. Backscattering-spectroscopy determination of oxygen supply is based on the principle that a light sent through a tissue at certain wavelengths is reflected by blood cells, depending on their haemoglobin content and oxygen saturation. Reflection angle decreases with increasing wavelength and this phenomenon favors Near Infrared (NIR) vs visible light to penetrate the tissue. NIR light wavelengths generally used are selected to be sensitive to deoxyhaemoglobin $(\mathrm{HHb}$, from 650 to 1000 $\mathrm{nm})$ and oxyhaemoglobin $\left(\mathrm{HbO}_{2}\right.$, peak between 700 and $1150 \mathrm{~nm}$ ) (9). Considering that most of the haemoglobin is located in the capillary and post-capillary system of the microvascular bed, measurements obtained mainly reflect the capillary-venous compartment of the microcirculation. Indexes obtained with NIRS technology are the result of the balance between $\mathrm{O}_{2}$ delivery and tissue oxygen consumption $\left(\mathrm{VO}_{2}\right)(9)$ and changes in NIRS light absorption reflect adjustments of oxygen transport at the microcirculation and its cellular utilization (10).

Previous studies highlighted that, in tendons, the oxidative metabolic rate is relatively low and that changes in blood volume and oxygen saturation can be detected by NIRS technology depending on training modalities (11-14). In particular, spectroscopic measurement using NIRS and red laser lights was performed to investigate the blood volume (total haemoglobin; $\mathrm{tHb}$ ) and oxygen saturation (oxygenated haemoglobin saturation; $\mathrm{StO}_{2}$ ) of the human Achilles tendon during single and repetitive isometric plantar flexions (15) and during short isometric voluntary isometric knee extensions in patellar tendon (16).

Tendons mechanical properties (cross sectional area, strain, stress, stiffness, Young's modulus) have been extensively investigated in physiological and pathological conditions (17-19), following diverse training modalities and in presence of tendinopathy. Overall, the available studies indicated that continous and simultaneous ultrasonography is accurate and reliable in assessing human tendon elongation and stiffness in all tested conditions including ramped isometric knee extensions as demonstrated in PT (16).

To obtain significant insight onto the relationship between tendon mechanical properties and oxygenation deterioration in promoting the onset of tendinopathy, technologies that can identify early changes in oxygen delivery and consumption, before the possible appearance of gross anatomical changes, including neoangiogenesis, may be of help. To this aim, the combination of ultrasounds and NIRS technologies represents an interesting future approach. Nevertheless, it is currently unknown, both in physiological and pathological conditions, whether a significant correlation exists between tendon anatomical and mechanical features (measured by US) and tendon/peritendon oxygen delivery and utilization (measured by NIRS), and to what extent changes in tendon physiological properties during contractions may be considered determinants of its oxygen delivery and utilization.

Thus, the present study investigated, for the first time, the correlation between PT mechanical properties and oxygenation (total hemoglobin $\mathrm{tHb}, \Delta[\mathrm{HHb}]$ and tissue oxygenation index $\left(\mathrm{TOI}=\mathrm{O}_{2} \mathrm{Hb} / \mathrm{tHb}\right)$ ) measured by NIRS during a single bout of isometric ramped knee extension contractions-relaxations in healthy men. Results indicated that tendon mechanical properties and tendon/ peritendon oxygen extraction and blood volume appears to be moderately correlated at least in PT and in absence of tendinopathy. 


\section{MATERIALS AND METHODS}

\section{Subjects}

Eight recreationally healthy active males with past experience (at least 2 years) in resistance exercise training (mean $\pm \mathrm{SD}$ : age $27 \pm 4 \mathrm{yrs}$; height $1.76 \pm 0.4 \mathrm{~m}$, mass $75 \pm 6.5 \mathrm{~kg}$ ) were recruited and completed the study. Exclusion criteria included the presence of a medical condition (musculoskeletal, respiratory, or cardiac). Subjects were fully informed of the aims, risks, and discomfort associated with the investigation. They provided their written informed consent to participate in this study, before completing a questionnaire to assess their health status. The study was conducted at the Sport Medicine Centre, University of Pavia, Voghera, Italy. During tests optimal conditions of temperature and humidity $\left(22^{\circ} \mathrm{C}\right.$ and $50 \%$ respectively) were present. The present study meets the ethical standards of the journal (20).

\section{Tendon mechanical properties}

Patellar tendon length, cross-sectional area (CSA), and elongation were measured using ultrasonography (CX30, Phillips) with the knee joint set at $90^{\circ}$ knee flexion (full extension $=0^{\circ}$ ) and the hip joint set at $85^{\circ}$ (supine $=$ $180^{\circ}$ ). The resting length, defined as the distance between the patella apex and the point at which the tendon inserts into the tibial tuberosity, was determined by positioning the 40-mm-wide, 4-12 $\mathrm{MHz}$ linear transducer in the sagittal plane over the PT and marking the location of the patella apex $(0 \%$ tendon length) and tibial tuberosity $(100 \%$ tendon length) on the skin with a permanent marker pen (figure 1) (21). Three more locations were then marked on the skin over the tendon $(25,50$, and $75 \%$ tendon length) to enable PT CSA to be measured from these axial images using Image Analysis Software (ImageJ V.1.45s, National Institute of Health, MD, USA). PT CSA measurements were normalized to body mass (21). Mechanical properties of the tendon were assessed by measuring the PT elongation during 2 maximal isometric (MVC, $4 \mathrm{~s}$ each) contractions and submaximal isometric contractions at 20, 40, 60, 80\% MVC. Before MVC, preconditioning of the tendon was obtained with a series of five submaximal isometric contractions. The linear transducer was then placed in the sagittal plane over the PT $(\sim 1 \mathrm{~cm}$ of the probe above the patella apex and $\sim 3 \mathrm{~cm}$ below). Ramped isometric contractions lasted $4 \mathrm{~s}$ with 3 min rest between each contraction. Visual feedback of force production was displayed on a screen in front of the participants to ensure that all contractions were performed at a constant loading rate. Trials were discarded when the torque trace deviated more than $10 \%$ from the required level. Unfortunately, in all subjects the 4 -cm-wide ultrasound transducer did not enable simultaneous scanning of both proximal and distal insertions of the PT, but this limitation did not affect the comparison between subjects.

\section{NIRS}

Tendon oxygenation was monitored during constant isometric contractions at 20-40-60-80\% MVC trials with portable continuous-wave NIRS device (Nimo, Nirox Brescia, Italy) for which the absorption characteristics of light at 780 and $850 \mathrm{~nm}$ depend on the relative oxygenation of $\mathrm{Hb}$ and myoglobin and the sum of absorbencies at the 2 wavelengths gives the change in local blood volume that is attributed to the change in total haemoglobin volume $(\mathrm{tHb})$ (22). Skinfold thickness at the site of application of the NIRS probe was determined before the exercise protocol by ultrasound: the measured average values of skin and subcutaneous tissue measured at $90^{\circ}$ knee flexion was 0.42 $\pm 0.04 \mathrm{~cm}$ at $25 \%$ length, $0.48 \pm 0.04 \mathrm{~cm}$ at $50 \%$ length, and $0.5 \pm 0.07 \mathrm{~cm}$ at $75 \%$ length. The NIRS probe composed of
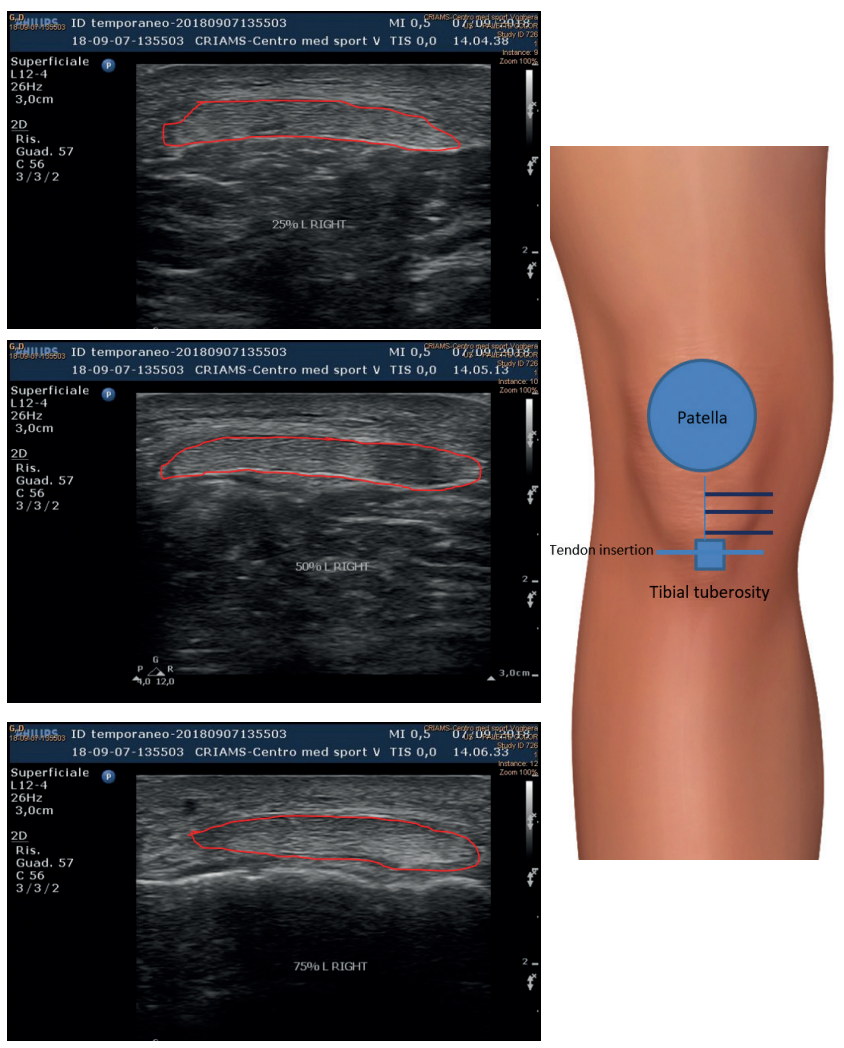

Figure 1. Axial ultrasound scans and measurements of the patellar tendon at $25 \%, 50 \%$ and $75 \%$ of length calculated between the patella apex and the tibial tuberosity. On the right schematic depiction of the axial positioning of the ultrasound probe. 
an emitter and detector pair, was firmly placed on the skin between previously pen marked patella apex $(0 \%$ tendon length) and tibial tuberosity (100\% tendon length) (figure 1) and secured with a small belt of Velcro straps. Elastic bandages were put around the muscle probe to prevent contamination from localised light. Pen-marks were made over the skin to indicate the margins of the plastic spacer in order to check for any downward sliding of the probe during tasks. Once secured in place, no sliding of the probe was observed. The probe was connected to a personal computer for data acquisition, A/D conversion, and subsequent analysis. Data were recorded at $2 \mathrm{~Hz}$. Variation of total hemoglobin $(\Delta[\mathrm{tHb}]), \Delta[\mathrm{HHb}]$, and tissue oxygenation index ( $\mathrm{TOI}=\mathrm{O}_{2} \mathrm{Hb} / \mathrm{tHb} \%$ ) from the baseline value were recorded after a 30 min light warm up and as a function of time during isometric tasks. Therefore, decreases/ increases in $\Delta[\mathrm{tHb}]$ were interpreted as evidence of changes in blood flow, increases in $\Delta[\mathrm{HHb}]$ and the decrease in TOI were interpreted as evidence of relative tendon deoxygenation and, conversely, as evidence of improved oxygenation.

\section{Analysis of tendon data}

PT elongation was recorded as the distance the patella apex moved from an identified reference point at every \% MVC. The video frame that corresponded to each contraction was exported as a portable network graphics (.png) file and the distance from the patella apex to the reference point was measured using image analysis software. Individual force-elongation curves were fitted with a second-order polynomial $\left(R^{2}>0.98\right.$ in all cases). Tendon stiffness ( $\Delta$ Force/ $\Delta \mathrm{PT}$ length) values were obtained at $80 \% \mathrm{MVC}$. At this \% MVC PT elongation was calculated as the change in tendon length from resting length. Tendon strain was calculated as the change in tendon length relative to the original tendon length $\left(\Delta L / L_{\mathrm{o}}\right)$, and expressed as a percentage. Young's modulus $(E)$ was calculated by multiplying stiffness $(k)$ with the ratio of the resting tendon length $\left(l_{0}\right)$ to mean tendon CSA, i.e., $E=k \times\left(l_{0} / s\right)$.

\section{STATISTICAL ANALYSES}

The mean and standard deviation $(s)$ were calculated for all variables. Data were tested for normality using the ShapiroWilks normality test. Pearson's correlations were used to determine relationships between patellar tendon mechanical properties and oxygenation. Pearson's correlation was used to compare significant differences between hemodynamics and myoelectric parameters according to Evans (20) (Pearson's $\mathrm{r}=0.00-0.19$, very weak; $r=0.20-0.39$, weak; $r$ $=0.40-0.59$, moderate; $r=0.60-0.79$, strong; $r=0.80-1.00$, very strong correlation). Statistical analysis was completed using Graphpad 5 (San Diego, California) and the significance level was set at $P \leq 0.05$.

\section{RESULTS}

Tendon properties are reported in table I. Correlation between \% MVC and elongation was fitted with a second order polynomial $\left(\mathrm{R}^{2}=0.99\right)$ (figure 2$)$. At all \% of MVC, a reduction of $\mathrm{tHb}$, and $\mathrm{TOI} \%$ and an increase of $\mathrm{HHb}$ was observed (figure 3 and table II), thus suggesting that an improved oxygen extraction during contraction counteracted the reduction of blood volume delivery. Interestingly, correlations between \% MVCs, $\Delta \mathrm{tHb}$ and $\Delta \mathrm{TOI} \%$ fitted with a second grade polynomial $\left(\mathrm{R}^{2}=0.94\right.$ and $\mathrm{R}^{2}=0.95$ respectively) (figure 4) and highlighted an almost constant value of oxygen extraction at low level MVC percentages (20-60\% MVC) (figure 4). Pearson's correlations between tendon mechanical properties and oxygenation parameters

Table I. Patellar tendon morphological and mechanical properties calculated in the whole cohort of subjects $(n=8)$; mean \pm SD. CSA, cross-sectional area; sCSA, cross-sectional area isometrically scaled to body mass0.67; MVC, maximal voluntary contraction; $\mathrm{MPa}$, megapascals; $\mathrm{GPa}$, gigapascals.

\begin{tabular}{lc}
\hline Tendon property & $109 \pm 4$ \\
\hline Mean CSA $\left(\mathrm{mm}^{2}\right)$ & $6.16 \pm 0.31$ \\
\hline Mean sCSA $\left(\mathrm{mm}^{2} / \mathrm{kg}^{0.67}\right)$ & $56 \pm 3$ \\
\hline Resting tendon length $(\mathrm{mm})$ & $1420 \pm 115$ \\
\hline Stiffness $(\mathrm{N} / \mathrm{mm})$ & $5.06 \pm 0.24$ \\
\hline Elongation $(\mathrm{mm})$ & $9.07 \pm 0.6$ \\
\hline Strain $(\%)$ & $65.7 \pm 3$ \\
\hline Stress $(\mathrm{MPa})$ & $0.72 \pm 0.1$ \\
\hline Young's modulus $(\mathrm{GPa})$ & \\
\hline
\end{tabular}

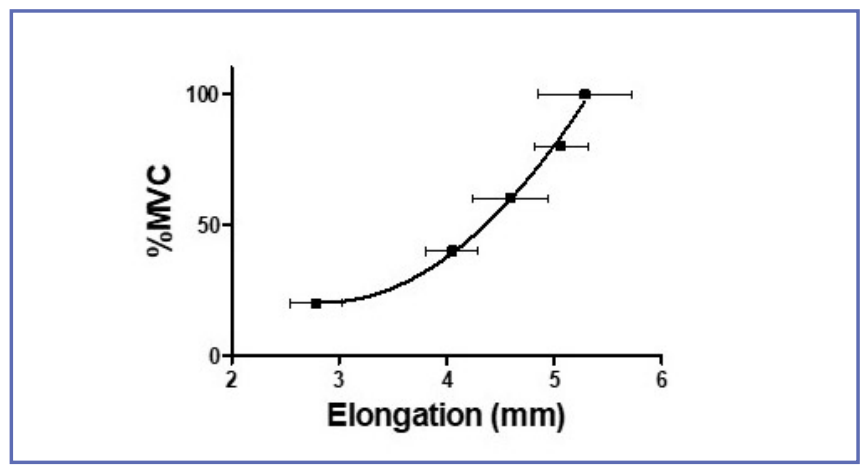

Figure 2. Non linear correlation between patellar tendon elongation and \%MVC fitted with a second order polinomial. 


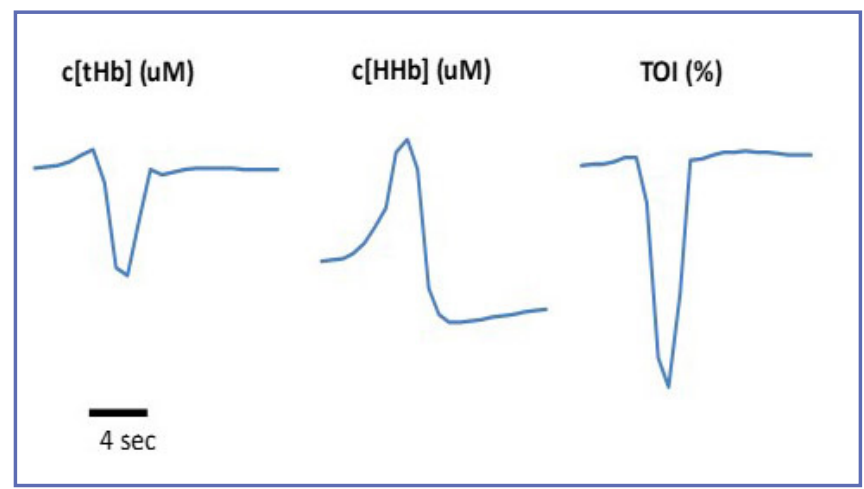

Figure 3. Example tracks describing change in $\mathrm{HHb}(\mathrm{mM})$, $\mathrm{TOI} \%$, tHb $(\mathrm{mM})$ in the patellar tendon during $4 \mathrm{sec}$ maximal voluntary contraction (MVC).

Table II. Patellar/peripatellar $\Delta \mathrm{tHb}, \Delta \mathrm{HHb}, \mathrm{TOI} \%$ measured during short $4 \mathrm{~s}$ duration ramped isometric contractions. $\mathrm{MVC}$, maximal voluntary contraction; $\Delta \mathrm{tHb}$, variation of total hemoglobin; $\triangle \mathrm{HHb}$, variation of deoxygenated hemoglobin; TOI, tissue oxygenation index.

\begin{tabular}{llll}
\hline MVC $\%$ & $\Delta \mathbf{t H b}$ & $\Delta \mathbf{H H b}$ & TOI $\%$ \\
\hline 20 & $-5.9 \pm 0.5$ & 1.234 & $-25.2 \pm 1.8$ \\
\hline 40 & $-6.1 \pm 0.6$ & $2.2 \pm 0.4$ & $-25 \pm 1$ \\
\hline 60 & $-5.1 \pm 1.9$ & $2.1 \pm 0.2$ & $-25.2 \pm 1.4$ \\
\hline 80 & $-8.2 \pm 0.56$ & $2.25 \pm 0.3$ & $-30.6 \pm 1.8$ \\
\hline 100 & $-12.9 \pm 0.9$ & $2.1 \pm 0.2$ & $-56.3 \pm 1.6$ \\
\hline
\end{tabular}

were analysed at $80 \%$ MVC. As regards oxygen extraction, $\Delta \mathrm{TOI} \%$ appeared negatively correlated with strain $(\mathrm{R}=$ - 0.64) and elongation $(R=-0.48)$, whereas a significant moderate positive correlation was found between Young modulus and stress and $\Delta \mathrm{TOI} \%(\mathrm{R}=0.69$ and $\mathrm{R}=0.62$ respectively). As regards blood volume, a negative correlation was found between elongation and $\Delta \mathrm{tHb}(\mathrm{R}=-0.44)$ and a positive correlation was found between stress and $\Delta \mathrm{tHb}(\mathrm{R}=0.4)$ (figure 5). No significant correlations were found between $\Delta \mathrm{tHb}$ and strain $(\mathrm{R}=-0.02)$ or Young modulus $(\mathrm{R}=0.19)$.

\section{DISCUSSION}

In the present study ultrasounds and NIRS were used to investigate whether mechanical properties of a human tendon correlate with the level of tendon/peritendon blood volume and oxygen extraction during ramped isometric extensions in healthy young subjects. Patellar tendon was chosen both because of its frequent involvement in overload injuries and for its easy accessibility to both techniques.

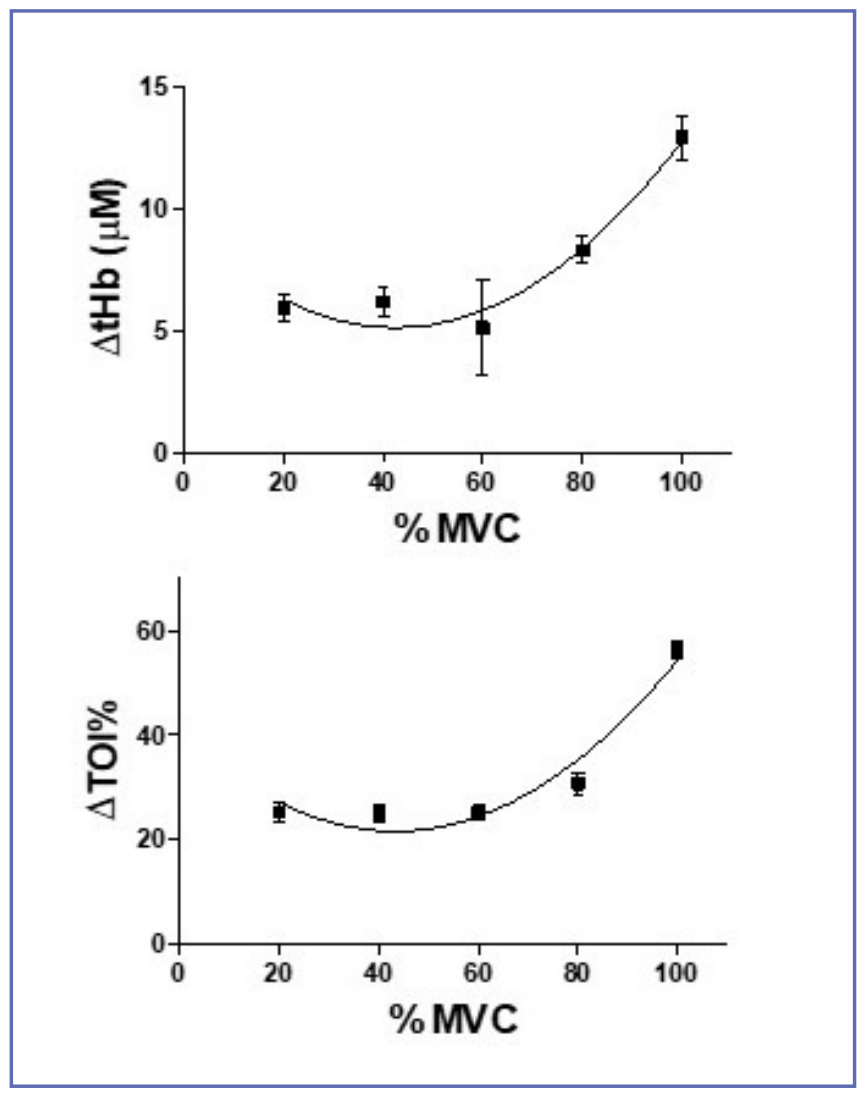

Figure 4. Correlations between blood volume $(\Delta t \mathrm{Hb})$ and deoxygenation ( $\triangle \mathrm{TO} \%$ ) vs \% MVC fitted with a second order polynomial.

The main findings were: 1) a non linear change in oxygen extraction and blood volume with increasing \% MVC; 2) a significant correlation between mechanical properties and blood flow and oxygen saturation $(\Delta \mathrm{TOI} \%)$ calculated during single bout at $80 \%$ MVC; 3 ) the absence of functional hyperemia at all \% MVC tested.

The observed reduction of blood volume and oxygen saturation are in agreement to previously published data on AT by Kubo et al. In particular, they used NIRS and red laser lights to investigate the blood volume (total haemoglobin; $\mathrm{tHb}$ ) and oxygen saturation (oxygenated haemoglobin saturation; $\mathrm{StO}_{2}$ ) of the human AT during single and repetitive isometric plantar flexions (15). In accordance with our data, results indicated a decrease in $\mathrm{tHb}$ and $\mathrm{StO}_{2}$ during a single bout of exercise, whereas an increase in blood volume and oxygen saturation was observed after repetition bouts. In other subsequent studies Kubo and collaborators $(15,16)$ investigated blood volume, oxygen saturation and associated stiffness changes induced by repeated isometric plantar flexions of short and long durations at $70 \%$ and $50 \%$ 


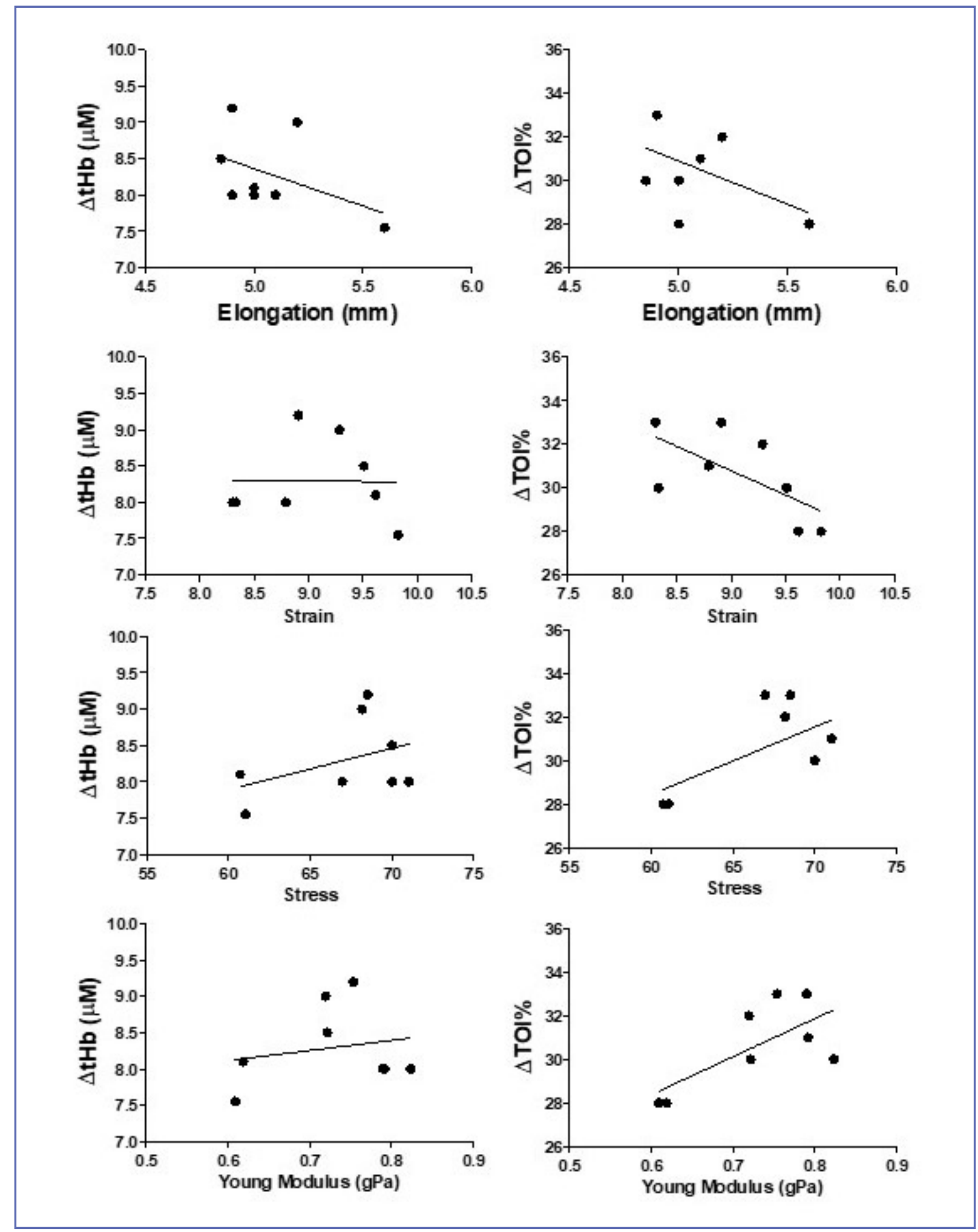

Figure 5. Pearson's correlations between patellar tendon mechanical properties (elongation, strain, stress and Young modulus) and change in blood volume $(\Delta \mathrm{tH} b)$ and deoxygenation $(\triangle \mathrm{TO} \%)$ at $80 \% \mathrm{MVC}$. 
MVC in AT and during short isometric 50\% MVC voluntary isometric knee extensions in PT. Importantly, results indicated an overall higher change in oxygen saturation and blood flow in PT compared to Achilles tendon. Interestingly, in our experimental conditions, a non linear increase in oxygen consumption (estimated as oxygen saturation) and decrease of blood volume of the patellar tendon was observed during a single bout of ramped isometric contractions suggesting that the tendon mechanical properties i.e. the tendon elongation at a given \% MVC, shape the tendon $\mathrm{tHb}$ and oxygenation status. Accordingly, at $80 \% \mathrm{MVC}$, a negative correlation was found between oxygen extraction $(\Delta \mathrm{TOI} \%) /$ blood volume and tendon deformation (strain and elongation) and a positive correlation was found between these parameters and stress and Young modulus. Oxygen extraction correlated with the tendon mechanical properties with higher significance than blood volume thus suggesting that oxygen extraction may represent the preferential mechanism with respect to vasodilation in satisfying the metabolic needs of the tendon during isometric contraction at high percentage of MVC.

In agreement with previous studies (16) in which single bouts of isometric knee extentions were performed, in our experimental conditions, during short lasting ramped isometric knee extensions, no functional hyperemia was found in all subjects tested, as demonstrated by concomitant reduction of $\mathrm{tHB}$ and TOI\% (not shown).

\section{LIMITATIONS OF THE STUDY}

This study has limitations. An important methodological issue is whether the measured $\mathrm{tHb}$ and $\Delta \mathrm{TOI} \%$ of the PT truly reflected the oxidative metabolic activity of the tendon. Concerning this point, we confirmed that the depth of the patellar tendon from the skin was consistent with the penetration depth of the NIR light. Using ultrasonography, we observed that the depth of the patellar tendon from the skin was $0.42 \pm 0.04 \mathrm{~cm}$ at $25 \%$ length, $0.48 \pm 0.04 \mathrm{~cm}$ at $50 \%$ length, and $0.5 \pm 0.07 \mathrm{~cm}$ at $75 \%$ length. Considering

\section{REFERENCES}

1. Maffulli N, Oliva F, Loppini M, Aicale R, Spiezia F, King JB. The Royal London Hospital Test for the clinical diagnosis of patellar tendinopathy. MLTJ 2017;7:315-322.

2. Millar NL, Reilly JH, Kerr SC, et al. Hypoxia: a critical regulator of early human tendinopathy. Ann Rheum Dis 2012;71:302-10

3. Longo UG, Oliva F, Denaro V, Maffulli N. Oxygen species and overuse tendinopathy in athletes. Disabil Rehabil 2008;30:1563-71. that, in our experimental conditions, the interdiode distance between the light source and photodetector allows an investigation depth of $15-20 \mathrm{~mm}$, and that the adipose tissue pad is a constant structure (bounded superiorly by the inferior pole of the patella, inferiorly by the anterior tibia, intermeniscal ligament, meniscal horns and infrapatellar bursa, anteriorly by the patellar tendon and posteriorly by the femoral condyles and intercondylar notch (21)), we may suppose that most of the signal we could measure probably derives from the patellar tendon. A second important methodological issue was that tendon mechanical properties were calculated based on ramped MVC and not on tendon MVC as antagonist coactivation and the tendon lever arm were not calculated. Lastly, the limited number of subjects mitigates the statistical significance of the correlation analysis.

\section{CONCLUSIONS}

Data obtained in this study suggest that continous NIRS technology can be used in combination with US to study patellar tendon mechanical properties, blood volume and oxygen utilization in healthy young subjects during isometric knee extensions. In particular, at $80 \%$ MVC a negative correlation was found between tendon elongation, measured by US, and oxygen extraction/blood flow, measured by NIRS, thus identifying this level of contraction as ideal for future studies on the possible overload-induced deterioration of tissue oxygenation virtually leading to subsequent structural and functional changes in the tendons.

\section{ACKNOWLEDGEMENTS}

English writing assistance was provided by Maria Douvli Smith (MD). This study was supported by crowdfunding \#Sport4Therapy to GD.

\section{CONFLICT OF INTERESTS}

The authors declare that they have no conflict of interests.

4. Maffulli N, Ewen SW, Waterston SW, et al. Tenocytes from ruptured and tendinopathic achilles tendons produce greater quantities of type III collagen than tenocytes from normal achilles tendons. An in vitro model of human tendon healing. Am J Sports Med 2000;28:499-505.

5. Alfredson H, Öhberg L. Increased intratendinous vascularity in the early period after sclerosing injection treatment in Achilles tendinosis. A healing response? Knee Surg Sports Traumatol Arthrosc 2006;14:399-401. 
6. Knobloch K. The role of tendon microcirculation in Achilles and patellar tendinopathy. J Orthop Surg Res 2008; 3: 18.

7. Astrom M, Svensson H. Tendon blood flow assessed by laser Doppler flowmetry. Scand J Plast Reconstr Surg Hand Surg 1991;25:213-5.

8. Knobloch K, Kraemer R, Lichtenberg A, et al. Achilles tendon and paratendon microcirculation in midportion and insertional tendinopathy in athletes. Am J Sports Med 2006;34:92-7.

9. Boushel R, Langberg H, Olesen J, et al. Regional blood flow during exercise in humans measured by near-infrared spectroscopy and indocyanine green. J Appl Physiol (1985) 2000;89:1868-78.

10. Duhaylongsod FG, Griebel JA, Bacon, DS, Wolfe WG. Piantadosi, C.A. 1993. Effects of muscle contraction oncytochrome a,a3 redox state. J Appl Physiol 75;790-97.

11. Langberg H, Bulow J, Kjaer M. Standardized intermittent static exercise increases peritendinous blood flow in human leg. Clin Physiol 1999;19:89-93.

12. Kubo K, Kanehisa H, Fukunaga T. Effects of different duration isometric contractions on tendon elasticity in human quadriceps muscles. J Physiol 2001;536:649-55.

13. Kubo K, Morimoto M, Komuro T, et al. Effects of plyometric and weight training on muscle-tendon complex and jump performance. Med Sci Sports Exerc 2007;39:1801-10.

14. Kubo K, Ohgo K, Takeishi R, et al. Effects of isometric training at different knee angles on the muscle-tendon complex in vivo. Scand J Med Sci Sports 2006;16:159-67.

15. Kubo K, Ikebukuro T, Tsunoda N, Kanehisa H. Changes in oxygen consumption of human muscle and tendon following repeat muscle contractions. Eur J Appl Physiol 2008;104:859-66.
16. Kubo K, Ikebukuro T. Blood circulation of patellar and Achilles tendons during contractions and heating. Med Sci Sports Exerc 2012;44:2111-7.

17. Couppé C, Suetta C, Kongsgaard M, Justesen L, Hvid LG, Aagaard P, Kjær M, Magnusson SP. The effects of immobilization on the mechanical properties of the patellar tendon in younger and older men. Clin Biomech (Bristol, Avon). 2012 Nov;27(9):949-54.

18. Kubo K, Tabata T, Ikebukuro T, Igarashi K, Yata H, Tsunoda N. Effects of mechanical properties of muscle and tendon on performance in long distance runners. Eur J Appl Physiol 2010;110(3):507-14.

19. Hansen P, Bojsen-Moller J, Aagaard P, Kjær M, Magnusson SP. Mechanical properties of the human patellar tendon, in vivo. Clin Biomech 2006;21(1):54-8.

20. Padulo J, Oliva F, Frizziero A, Maffulli N. Muscle, Ligaments and Tendons Journal-Basic principles and recommendations in clinical and field science research: 2016 update. MLTJ 2016;6:1-5.

21. Helland C, Bojsen-Møller J, Raastad T, et al. Mechanical properties of the patellar tendon in elite volleyball players with and without patellar tendinopathy. Br J Sports Med 2013;47:862.

22. Zampella C, Calanni L, Barbero M, et al. Correlations between myoelectric and hemodynamic parameters changes in biceps brachii during sustained isometric contraction in healthy elderly. J Sci Sports Exerc 2019;1:116-123.

23. Evans JD. Straightforward statistics for the behavioral sciences. Pacific Grove: Brooks/Cole Publishing; 1996.

24. Gallagher J, Tierney P, Murray P, O'Brien M. The infrapatellar fat pad: anatomy and clinical correlations. Knee Surg Sports Traumatol Arthrosc 2005;13:268-72. 